\title{
Isolated Gallbladder Perforation in Cases of Blunt Trauma Abdomen
}

\author{
Gupta MK ${ }^{1}$, Ahmad K ${ }^{1}$, Kumar A ${ }^{1}$, Santhalia PK ${ }^{1}$, Joshi BR ${ }^{2}$, Rauniyar RK ${ }^{1}$ \\ ${ }^{1}$ Department of Radiodiagnosis and Imaging, ${ }^{2}$ Department of Surgery, B.P. Koirala Institute \\ of Health Sciences (BPKIHS), Dharan, Nepal
}

\begin{abstract}
Isolated perforation of gallbladder (GB) following blunt trauma abdomen is extremely rare and may pose a diagnostic challenge. Early diagnosis and treatment is of paramount importance to reduce morbidity and mortality associated with GB perforation. We present two cases of isolated GB perforation in patients of blunt abdominal trauma diagnosed on multi-detector computed tomography (MDCT) which was corroborated by bile stained peritoneal aspirate and subsequently confirmed by surgery. Both the patients underwent open cholecystectomy with good outcome.
\end{abstract}

Keywords: Blunt abdominal trauma, Isolated gallbladder perforation, Multi-detector computed tomography.

\section{Introduction}

GB is partly surrounded by liver, cushioned by omentum and intestine and shielded by rib cage. Due to this relatively protected anatomic position, GB is frequently sheltered from injury in abdominal trauma. ${ }^{1,2}$ Traumatic injuries of GB are usually associated with damage to other abdominal organs and isolated GB injury is exceedingly rare. Patients with isolated GB injury are at increased risk for significant morbidity and mortality due to non-specific presentation resulting in delayed diagnosis. Two percent of patients who undergo laparotomy for

Correspondence to: Dr. Mukesh Kumar Gupta, Associate Professor, Department of Radiodiagnosis and Imaging, B.P. Koirala Institute of Health Sciences, Dharan, Nepal. E-mail: mukeshgupta148@yahoo.com abdominal trauma are found to have GB injury. The spectrum of GB injuries includes contusion, laceration, and avulsion. ${ }^{1}$ Preoperative diagnosis can be made effectively with MDCT allowing early treatment of the cases which is of paramount importance to reduce complications associated with GB perforation. We present a series of two cases with pre-operative MDCT diagnosis of isolated traumatic GB perforation treated successfully with open cholecystectomy.

\section{Case Report}

\section{Case 1}

A 49-year-old male presented with progressive abdominal pain and distension after 3 days of blunt abdominal trauma caused by fall on the floor. Physical examination revealed generalized abdominal 


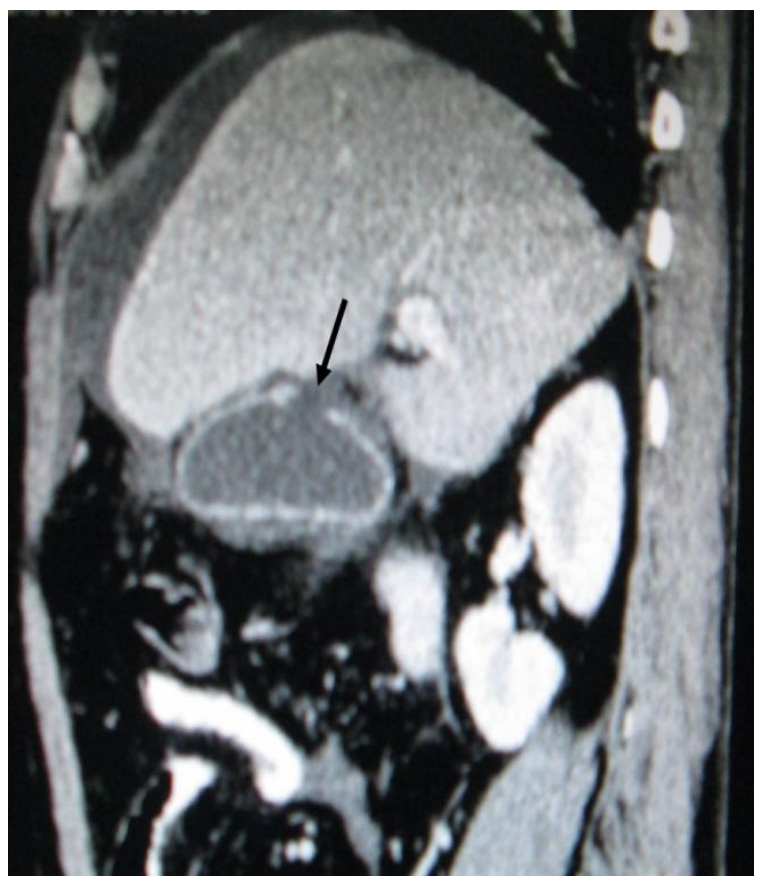

Fig. 1: Sagittal reformatted contrast enhanced MDCT image (Case 1) shows a focal defect (arrow) in the GB wall, minimal pericholecystic fluid collection, peritoneal fluid collection predominantly in perihepatic space and enhancement of thickened peritoneum, suggesting biliary peritonitis from GB perforation.

tenderness and some guarding. Patient's vitals were stable and the blood tests were within normal limits. Contrast enhanced MDCT (Fig.1) with multi planar reconstruction of the data revealed a focal defect in the GB wall in the body region, peritoneal fluid collection predominantly in perihepatic space and enhancement of thickened peritoneum, suggesting biliary peritonitis from GB perforation. Rest of the intra-abdominal organs revealed no significant findings on CT scan. Diagnostic peritoneal aspirate yielded bile stained fluid. Subsequent laparotomy confirmed isolated GB perforation and the patient was successfully treated with cholecystectomy. The post-operative course was unremarkable and the patient had good clinical recovery.
Case 2

A 65-year-old male presented with abdominal pain and distension after 2 days of blunt abdominal trauma following motor vehicle accident. Physical examination showed tenderness in right hypochondrium. Patient's vitals were stable and the blood tests were within normal limits. A high index of clinical suspicion was maintained for internal injury and CT scan of the abdomen was performed. Contrast enhanced MDCT (Fig. 2) with multiplanar reconstruction of the data revealed a focal defect in GB wall, shrunken GB and large peritoneal fluid collection, suggesting GB perforation. No other intra-abdominal injuries were identified on CT scan. Diagnostic peritoneal aspirate yielded bile stained fluid. Isolated GB perforation was confirmed at subsequent laparotomy and the patient was successfully managed with cholecystectomy. The postoperative course was uneventful and the patient had good clinical recovery following surgery.

\section{Discussion}

GB perforation in cases of blunt trauma abdomen is extremely rare because of its relatively protected anatomical position. Gallbladder injury occurs in 1.9-2.1\% of cases of blunt abdominal trauma. ${ }^{1}$ Isolated GB injury, as in our case, is far less common. In a study by Soderstrom et $\mathrm{al}^{1}$ on 31 patients with injury to GB, only one demonstrated isolated GB injury. Wiener et $\mathrm{al}^{3}$ reported isolated GB injury in 3 out of total 6 cases of GB injury. Most blunt traumatic GB injuries results from motor vehicle accident, fall, and kick or blow to the abdomen. The first case in our series sustained trauma from fall on the floor and 


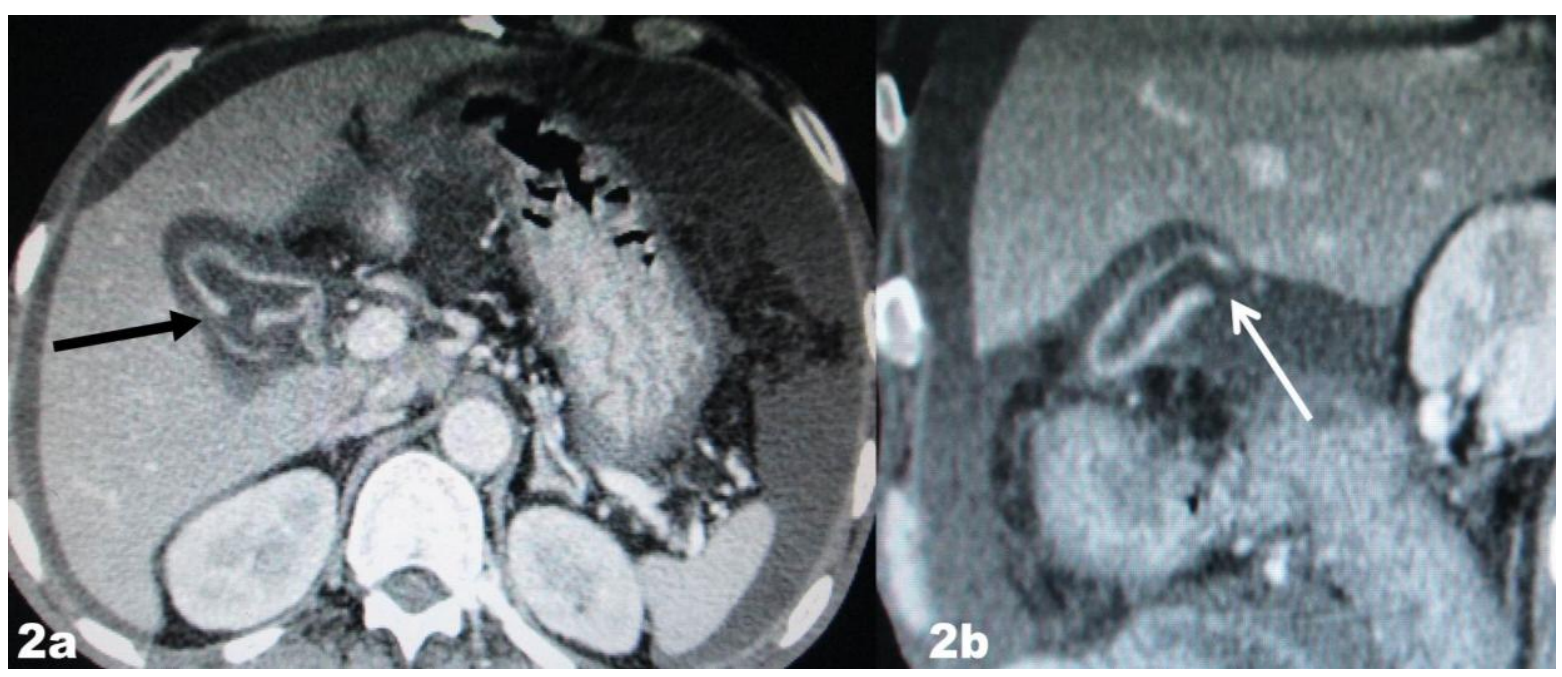

Fig. 2: Axial (2a) and sagittal reformatted (2b) contrast enhanced MDCT images (case 2) shows a focal defect (arrows) in GB wall, shrunken GB and pericholecystic and peritoneal fluid collection, suggesting GB perforation.

the second case had trauma from motor vehicle accident. Factors predisposing to blunt GB injuries are thin-walled normal gallbladder, distended gallbladder such as in fasting state, and alcohol consumption that increases the tone of sphincter of Oddi and biliary tract pressure. ${ }^{4,5}$ On the other hand, a thickened or diseased gallbladder wall, secondary to chronic inflammation, is protective. ${ }^{2,6,7}$ Injuries to the gallbladder can be classified as contusion, avulsions, and laceration. Laceration (also referred as ruptures or perforation) is the most commonly reported blunt traumatic GB injury. ${ }^{1}$

The diagnosis of GB perforation following blunt abdominal trauma is mostly incidental at laparotomy performed for evaluation and treatment of other visceral injuries. The preoperative diagnosis of blunt traumatic GB perforation is challenging which may lead to delayed diagnosis with increased risk of morbidity and mortality. The clinical features can be variable, nonspecific and misleading. ${ }^{1,5}$ Aspiration of bilious peritoneal fluid may aid in the diagnosis of blunt traumatic GB injury, however negative taps have also been reported and false positive result can occur from injury to biliary tree, liver and upper GI tract. Diagnostic peritoneal lavage, although a useful diagnostic tool in the evaluation of blunt abdominal trauma, has limited role particularly in detection of GB injury. ${ }^{1,5}$

Radiological imagings play important role in pre-operative diagnosis of GB injury as well as concomitant injury to the other organs. MDCT with acquisition of thinner images and multiplanar reconstruction of the data allows better delineation of the GB injury. CT findings associated with gallbladder perforations can include pericholecystic fluid collection, collapsed GB, intraluminal hyperdense blood, GB wall defect, thickening of wall, mass effect on the adjacent organs such as liver, duodenum or right kidney due to distension of $\mathrm{GB}$, and streaky omentum or mesenteric fat. ${ }^{6,8-10}$ Preoperative MDCT showed defect in the GB wall along with pericholecystic and peritoneal fluid collection in both of the cases in our series. Ultrasound can be 
effective in the diagnosis of GB injury as well, and the findings may include complex echogenic pericholecystic fluid collection, thickened and edematous GB wall, collapsed lumen despite prolonged fasting, and disruption of GB wall with focal loss of its reflectivity. ${ }^{8,11}$ Although ultrasound is superior for evaluation of non-traumatic GB pathology, CT is more accurate and effective for traumatic injuries in identification of high density hemorrhagic fluid or fluid/fluid level within GB lumen, and better characterization of concomitant adjacent organ injury as well. ${ }^{10}$ MRI and hepatobiliary scintigraphy are other possible diagnostic modalities in equivocal cases. Cholecystectomy is now the treatment of choice in GB perforation. Both of our cases underwent open cholecystectomy and had good outcome.

\section{Conclusion}

Isolated blunt traumatic GB perforation is exceedingly rare owing to its relatively protected anatomic location and may pose a diagnostic challenge resulting in delayed diagnosis with increased risk of significant morbidity and mortality. MDCT with multiplanar reconstruction of the data is the effective noninvasive tool for early diagnosis of GB perforation. Bile stained peritoneal aspirate help to corroborate the imaging findings in equivocal cases. Cholecystectomy is the treatment of choice in GB perforation.

\section{References}

1. Soderstrom CA, Maekawa K, DuPriest RW, Cowley RA. Gallbladder injuries resulting from blunt abdominal trauma: an experience and review. Ann Surg 1981;193(1):60-6.
2. Schachter P, Czerniak A, Shemesh E, Avigad I, Lotan G, Wolfstein I. Isolated gallbladder rupture due to blunt abdominal trauma. HPB Surgery 1989;1(4):359-62.

3. Wiener I, Watson LC, Wolma FJ. Perforation of the gallbladderdue to blunt abdominal trauma. Arch surg 1982;117(6):805-7.

4. Chen X, Talner LB, Jurkovich GJ. Gallbladder avulsion due to blunt trauma. Am J Roentgenol 2001;177(4):822.

5. Sharma O. Blunt gall bladder injuries: presentation of twenty-two cases with review of the literature. $\mathbf{J}$ Trauma 1995;39(3):576-80.

6. Jaggard MK, Johal NS, Choudhry M. Blunt abdominal trauma resulting in gallbladder injury: a review with emphasis on pediatrics. J Trauma 2011;70(4):1005-1010.

7. Spigos DG, Tan WS, Larson G, Palani C, Zaintoon MM, Capek V. Diagnosis of traumatic rupture of the gallbladder. Am J Surg 1981;141(6):731-735.

8. Kim PN, Lee KS, Kim IY, Bae WK, Lee BH. Gallbladder perforation: comparison of US findings with CT. Abdom Imaging 1994;19(3):239-242.

9. Erb RE, Mirvis SE, Shanmuganathan K. Gallbladder injury secondary to blunt trauma: CT findings. J Comput Assist Tomogr 1994;18(5):778-784. 
10. Jeffrey RB Jr, Federle MP, Laing FC, 11. Siskind BN, Hawkins HB, Cinti DC, Wing VW. Computed tomography of Zeman RK, Burrell MI. Gallbladder blunt trauma to the gallbladder. J Comput Assist Tomogr 1986;10(5):756-758. perforation: an imaging analysis. J Clin Gastroenterol 1987;9(6):670-678. 